\title{
Bipartite graphs with five eigenvalues and pseudo designs
}

\author{
Ebrahim Ghorbani
}

Received: 20 November 2010 / Accepted: 11 November 2011 / Published online: 3 December 2011 (C) Springer Science+Business Media, LLC 2011

\begin{abstract}
A pseudo $(v, k, \lambda)$-design is a pair $(X, \mathcal{B})$, where $X$ is a $v$-set, and $\mathcal{B}=$ $\left\{B_{1}, \ldots, B_{v-1}\right\}$ is a collection of $k$-subsets (blocks) of $X$ such that any two distinct $B_{i}, B_{j}$ intersect in $\lambda$ elements, and $0 \leq \lambda<k \leq v-1$. We use the notion of pseudo designs to characterize graphs of order $n$ whose (adjacency) spectrum contains zero and $\pm \theta$ with multiplicity $(n-3) / 2$ where $0<\theta \leq \sqrt{2}$. Meanwhile, partial results confirming a conjecture of $\mathrm{O}$. Marrero on a characterization of pseudo $(v, k, \lambda)$-designs are obtained.
\end{abstract}

Keywords Spectrum of graph · Pseudo design · BIBD · DS graph · Cospectral graphs $\cdot$ Incidence graph $\cdot$ Subdivision of star

\section{Introduction}

To study bipartite graphs with four/five distinct (adjacency) eigenvalues, one needs to investigate combinatorial designs with two singular values (i.e., the matrix $N N^{\top}$ has only two positive eigenvalues, where $N$ is the $(0,1)$-incidence matrix of the design). Recently, van Dam and Spence [12] studied bipartite graphs with four eigenvalues which are precisely the incidence graphs of designs with two singular values with nonsingular and square $N$. These designs, called uniform multiplicative designs, were introduced by Ryser [9]. In [13], bipartite biregular graphs with five distinct eigenvalues were investigated. These graphs correspond to designs with two singular values,

E. Ghorbani $(\bowtie)$

Department of Mathematics, K.N. Toosi University of Technology, P.O. Box 16315-1618,

Tehran, Iran

e-mail: e_ghorbani@ipm.ir

E. Ghorbani

School of Mathematics, Institute for Research in Fundamental Sciences (IPM),

P.O. Box 19395-5746, Tehran, Iran 
constant block size and constant replication number. These designs are called partial geometric designs, first introduced by Bose (see [1]). Designs with few distinct singular values are also of interest from statistical point of view. R.A. Bailey (cf. [3]) recently raised the question of which designs have three eigenvalues. To be more specific, it was asked for which designs with constant block size, constant replication, and incidence matrix $N$, does $N N^{\top}$ have three distinct eigenvalues.

In this paper, we continue this line by studying bipartite graphs with five eigenvalues where the second largest eigenvalue is relatively small. To be more precise, we characterize the graphs with $n$ vertices whose spectrum contains $\left\{0,( \pm \theta)^{\frac{n-3}{2}}\right\}$ with $0<\theta \leq \sqrt{2}$. The restriction to $\sqrt{2}$ comes from our limited knowledge of the corresponding designs. Having enough information of related designs, one can characterize the graphs with larger $\theta$. As it will be explained in the next section, it follows that $\theta$ must be a square root of an integer. So the next possible $\theta$ is $\sqrt{3}$.

The graphs with $n$ vertices whose spectrum contains $( \pm \theta)^{\frac{n-2}{2}}$ with $0<\theta \leq \sqrt{2}$ were already characterized by van Dam and Spence [12]. Note that the incidence graphs of symmetric $(v, k, \lambda)$-designs are precisely the regular graphs with the required property and $\theta=\sqrt{k-\lambda}$.

The graphs of the subject of the paper have a close connection with a family of combinatorial designs called pseudo designs. Therefore, we first study pseudo designs following Marrero [5, 6] and Woodall [14]. Our investigation has some implications on a conjecture of Marrero on a characterization of pseudo designs. We then make use of these results to determine the families of graphs whose spectrum contains $\left\{0,( \pm \theta)^{\frac{n-3}{2}}\right\}$.

By means of the spectral characterization of the aforementioned graphs, we find some new families of graphs which are DS (i.e., determined by spectrum). Finding new families of DS graphs is one of the challenging and very active research subjects in spectral graph theory. For more about DS graphs, see the surveys [10, 11].

\section{Preliminaries}

All the graphs we consider in this paper are finite, simple, and undirected. The order of a graph $G$ is the number of vertices of $G$. By the eigenvalues of $G$ we mean those of its adjacency matrix. The spectrum of $G$ is the multiset of eigenvalues of $G$. The subdivision of a graph $G$ is the graph obtained by inserting a new vertex on every edge of $G$. We denote by $\mathcal{S}_{2 k+1}$ the subdivision of the star $K_{1, k}$. The complete bipartite graph $K_{k, k}$ minus a perfect matching is denoted by $\mathcal{L}_{k, k}$. We denote by $\mathcal{H}_{k, k+1}$ the graph resulting from adding a new vertex to $\mathcal{L}_{k, k}$ and joining all vertices of one part of it to the new vertex. The adjacency matrix of a bipartite graph $G$ can be rearranged so that it has the form

$$
\left(\begin{array}{cc}
O & N \\
N^{\top} & O
\end{array}\right)
$$

with square zero matrices $O$. We denote by $J_{r, \ell}$ the all-1 matrix with $r$ rows and $\ell$ columns, and by $J_{r}$ if it is a square matrix. When the order of the matrix is clear from the context, we drop the subscripts. The matrix $N$ is called the bipartite adjacency matrix of $G$. The bipartite graphs of order $n=2 k+1$ with bipartite adjacency 

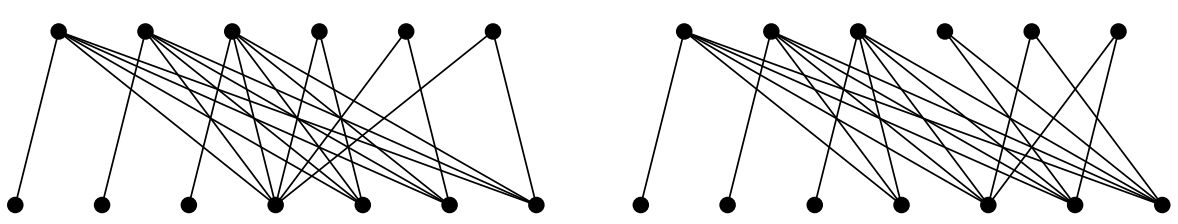

Fig. 1 The graphs $\mathcal{R}_{13}$ (left) and $\mathcal{Q}_{13}$ (right)

matrices

$$
\left(\begin{array}{cc}
I_{k-3} & J \\
O & \widetilde{I}_{3}
\end{array}\right)_{k \times(k+1)}, \quad\left(\begin{array}{cc}
\widetilde{I_{k-3}} & J \\
O & J_{3}-I_{3}
\end{array}\right)_{k \times(k+1)}
$$

are denoted by $\mathcal{R}_{n}$ and $\mathcal{Q}_{n}$, respectively, where $\widetilde{I}_{\ell}$ is the matrix resulting from extending the identity matrix by an all-1 column vector, i.e.,

$$
\tilde{I}_{\ell}=\left(I_{\ell} \mathbf{1}_{\ell}\right) \text {. }
$$

The graphs $\mathcal{R}_{13}$ and $\mathcal{Q}_{13}$ are depicted in Fig. 1.

A combinatorial design is a pair $(X, \mathcal{B})$, where $X$ is a set of points, and $\mathcal{B}$ is a collection of subsets of $X$, called blocks, together with an incidence relation between the points and the blocks. A balanced incomplete block design $\operatorname{BIBD}(b, v, r, k, \lambda)$ is a combinatorial design with $v$ points and $b$ blocks all of which have the same size $k$ and the incidence relation that any 2 -subset of $X$ is contained in exactly $\lambda$ blocks where $0 \leq \lambda$ and $k \leq v-1$. It follows that every element of $X$ is contained in a same number $r$ of blocks. Necessary conditions for the existence of a $\operatorname{BIBD}(b, v, r, k, \lambda)$ are

$$
\begin{gathered}
v r=b k, \\
r(k-1)=\lambda(v-1) .
\end{gathered}
$$

$\mathrm{A} \operatorname{BIBD}(b, v, r, k, \lambda)$ with $b=v$ (and so $r=k$ ) is called a symmetric $(v, k, \lambda)$ design. It is known that in a symmetric $(v, k, \lambda)$-design two distinct blocks $B_{i}, B_{j}$ $(1 \leq i, j \leq v)$ intersect in $\lambda$ elements. A pseudo $(v, k, \lambda)$-design is a pair $(X, \mathcal{B})$, where $X$ is a $v$-set, and $\mathcal{B}=\left\{B_{1}, \ldots, B_{v-1}\right\}$ is a collection of $k$-subsets (blocks) of $X$ such that any two distinct $B_{i}, B_{j}$ intersect in $\lambda$ elements, and $0 \leq \lambda<k \leq v-1$. Each combinatorial design is completely determined by its corresponding incidence matrix; this is the $(0,1)$-matrix $A=\left(a_{i j}\right)$ whose rows and columns are indexed by the blocks and the points of the design, respectively, where $a_{i j}=1$ if $x_{j} \in B_{i}$ and $a_{i j}=0$ if $x_{j} \notin B_{i}$. The incidence graph of a design $\mathcal{D}$ is a bipartite graph such that its bipartite adjacency matrix is the incidence matrix of $\mathcal{D}$.

Remark In order to avoid trivial cases, we assume that the designs considered in this paper (and so their incidence graphs) are connected. Therefore, the Perron-Frobenius theorem (cf. [4, p. 178]) can be applied. It follows that the largest singular value has multiplicity one and a positive eigenvector. Another consequence is that if $N$ is the bipartite adjacency matrix of a connected bipartite graph of order $n$ with five distinct eigenvalues where the zero eigenvalue is of multiplicity 1, then the characteristic polynomial of $N N^{\top}$ is of the form $x\left(x^{2}-a\right)^{\frac{n-3}{2}}\left(x^{2}-b\right)$. As mentioned in [12], it 
turns out that if $n>5$, then $a$ and $b$ must be integers. For $n=5$, there are only two such graphs with the following bipartite adjacency matrices:

$$
\left(\begin{array}{lll}
1 & 1 & 1 \\
0 & 0 & 1
\end{array}\right) \text { and }\left(\begin{array}{lll}
1 & 1 & 1 \\
0 & 1 & 1
\end{array}\right) \text {. }
$$

The spectra of these two graphs are $\{0, \pm \sqrt{2 \pm \sqrt{2}}\}$ and $\left\{0, \pm \frac{1}{2} \sqrt{10 \pm 2 \sqrt{17}}\right\}$, respectively. Therefore, if $0<\theta \leq \sqrt{2}$ and $\left\{0,( \pm \theta)^{\frac{n-3}{2}}\right\}$ is contained in the spectrum of a graph of order $n>5$, then $\theta=1$ or $\theta=\sqrt{2}$.

\section{Pseudo $(v, k, \lambda)$-designs}

Pseudo designs were studied by Marrero [5, 6] and Woodall [14]. Woodall used another terminology; he called pseudo designs near-square $\lambda$-linked designs. ${ }^{1}$ We follow the terminology of Marrero.

A pseudo $(v, k, \lambda)$-design is called primary if $v \lambda \neq k^{2}$ and is called nonprimary when $v \lambda=k^{2}$. In [7] it is shown that in a nonprimary pseudo design, $v=2 k$. Thus, a pseudo $(v, k, \lambda)$-design is nonprimary if and only if $v=4 \lambda$ and $k=2 \lambda$.

The existence of a nonprimary pseudo $(v, k, \lambda)$-design is equivalent to the existence of an Hadamard design:

Theorem 1 (Marrero-Butson [7]) The incidence matrix of a given pseudo $(4 \lambda, 2 \lambda, \lambda)$-design can always be obtained from the incidence matrix $A$ of a symmetric $(4 \lambda-1,2 \lambda-1, \lambda-1)$-design by adjoining one column of all $1 \mathrm{~s}$ to $A$ and then possibly complementing some rows of $A$.

In the theorem, complementing a row means that 0 s and $1 \mathrm{~s}$ are interchanged in that row. For example, take the Fano plane which is the unique symmetric $(7,3,1)$ design with points $\{1, \ldots, 7\}$ and blocks $\{124,235,346,457,156,267,137\}$. Now the theorem asserts that by adding a new point to all the blocks, namely 8 , and complementing any set of blocks we get a pseudo $(8,4,2)$-design. For example, if we do this for the first block, we have the pseudo design with blocks $\{3567,2358,3468,4578,1568,2678,1378\}$. For primary pseudo designs, we have the following:

Theorem 2 (Marrero [5, 6]) The incidence matrix A of a primary pseudo $(v, k, \lambda)$ design $\mathcal{D}$ can be obtained from the incidence matrix of a symmetric $(\bar{v}, \bar{k}, \bar{\lambda})$-design whenever $\mathcal{D}$ satisfies one of the following arithmetical conditions on its parameters.

(i) If $(k-1)(k-2)=(\lambda-1)(v-2)$, then $A$ is obtained by adjoining a column of $1 s$ to the incidence matrix of a symmetric $(v-1, k-1, \lambda-1)$-design.

(ii) If $k(k-1)=\lambda(v-2)$, then $A$ is obtained by adjoining a column of 0 s to the incidence matrix of a symmetric $(v, k, \lambda)$-design.

\footnotetext{
${ }^{1}$ A square $\lambda$-linked design consists of $v$ points and $v$ blocks such that any two distinct blocks intersect in $\lambda$ elements. This configuration is called a $\lambda$-design by Ryser [8] if in addition there exist two blocks of different sizes.
} 
(iii) If $k(k-1)=\lambda(v-1)$, then $A$ is obtained from discarding a row from the incidence matrix of a symmetric $(v, k, \lambda)$-design.

(iv) If $k=2 \lambda$, then $A$ is obtained from the incidence matrix $B$ of a symmetric $(v, k, \lambda)$-design as follows: a row is discarded from $B$ and then the $k^{\prime}$ columns of $B$ which had $a 1$ in the discarded row are complemented ( 0 s and $1 \mathrm{~s}$ are interchanged in these columns).

It was conjectured by Marrero [5, 6] that given a primary pseudo $(v, k, \lambda)$-design, "completion" or "embedding" between the given pseudo design and some symmetric $(\bar{v}, \bar{k}, \bar{\lambda})$-design is always possible. In other words:

Conjecture 3 (Marrero [5, 6]) The parameters of a given primary pseudo $(v, k, \lambda)$ design satisfy at least one of the four conditions of Theorem 2.

He proved the validity of his conjecture for $\lambda=1$.

Theorem 4 (Marrero [6], Woodall [14]) Let $A$ be the incidence matrix of a given primary pseudo $(v, k, \lambda)$-design, so that $A$ has two distinct column sums $s_{1}$ and $s_{2}$. Let $y=\left(k+\lambda(v-2)-k s_{2}\right) /\left(s_{1}-s_{2}\right)$, and let $f$ be the number of columns of $A$ having the column sum $s_{1}$. Then, after an appropriate permutation of the columns of $A$, it must be possible to write $A=\left[M_{v-1, f} N_{v-1, v-f}\right]$, where $M$ is the incidence matrix of $a \operatorname{BIBD}\left(\bar{b}=v-1, \bar{v}=f, \bar{r}=s_{1}, \bar{k}=y, \bar{\lambda}=s_{1}-k+\lambda\right)$, and $N$ is the incidence matrix of a $\operatorname{BIBD}\left(\bar{b}=v-1, \bar{v}=v-f, \bar{r}=s_{2}, \bar{k}=k-y, \bar{\lambda}=s_{2}-k+\lambda\right)$. (Note that $f$ may take the values 1 or $v-1$, too.)

In order to study the graphs of the subject of this paper, we need to characterize pseudo designs with $k-\lambda=1$ or 2 . For this, we need the following lemma.

Lemma 5 Let $\mathcal{D}$ be $a \operatorname{BIBD}(b, v, r, k, \lambda)$.

(i) If $r=\lambda+1$, then $\mathcal{D}$ is either the symmetric $(v, 1,0)$-design or the symmetric $(v, v-1, v-2)$-design.

(ii) If $r=\lambda+2$, then $\mathcal{D}$ is one of the $\operatorname{BIBD}(2 v, v, 2,1,0), \operatorname{BIBD}(6,4,3,2,1)$, $\operatorname{BIBD}(6,3,3,2,2)$, the symmetric $(7,3,1)$-design, or the symmetric $(7,4,2)$ design.

Proof Part (i) is straightforward. We prove (ii). First let $b>v$. So $\lambda+1=r-1 \geq k$. By (2), $(\lambda+2)(k-1)=\lambda(v-1)$. If $\lambda=0$, then $r=2$ and $k=1$. So, by (1), $b=2 v$, which means that $\mathcal{D}$ is $\operatorname{BIBD}(2 v, v, 2,1,0)$. If $\lambda=1$, then $r=3$, and so $v=3 k-2$. We have $k \neq 1$ since otherwise $v=1$, which is impossible. Thus $k=2$. It turns out that $\mathcal{D}$ is $\operatorname{BIBD}(6,4,3,2,1)$. If $\lambda=2$, then $r=4$, and so $v=2 k-1$. By $(1), b k=4(2 k-1)$, from which it follows that $k=2$ and thus $b=6$. Hence $\mathcal{D}$ is $\operatorname{BIBD}(6,3,3,2,2)$. Let $\lambda \geq 3$. If $\lambda$ is odd, then $\lambda \mid k-1$, and thus $\lambda=k-1$. If $\lambda$ is even, then $\frac{\lambda}{2} \mid k-1$. Since $\lambda \geq k-1$, it follows that either $k-1=\lambda$ or $k-1=\frac{\lambda}{2}$; the latter is impossible due to (2). Therefore $(k+1)(k-1)=(k-1)(v-1)$, so $v=k+2$. On the other hand, $b k=(k+1)(k+2)$, which is impossible since $k \geq 4$. Now let $b=v$. So $r=k=\lambda+2$. We have $(\lambda+2)(\lambda+1)=\lambda(v-1)$. Clearly $\lambda \neq 0$. 
If $\lambda=1$, then $k=3$ and $v=7$. So $\mathcal{D}$ is the symmetric $(7,3,1)$-design. If $\lambda=2$, then $k=4$ and $v=7$. So $\mathcal{D}$ is the symmetric $(7,4,2)$-design.

Theorem 6 Let $\mathcal{D}$ be a pseudo $(v, k, \lambda)$-design.

(i) If $k=\lambda+1$, then $\mathcal{D}$ is obtained from the symmetric $(v-1,1,0)$-design or the symmetric $(v-1, v-2, v-3)$-design by either adding an isolated point or a point which belongs to all of the blocks.

(ii) If $k=\lambda+2$, then, up to isomorphism, $\mathcal{D}$ is one of the $\mathcal{D}_{i}=\left(\{1, \ldots, 8\}, \mathcal{B}_{i}\right)$, $i=1,2,3,4$, where

$$
\begin{aligned}
& \mathcal{B}_{1}=\{1238,1458,1678,3568,2478,3468,2568\}, \\
& \mathcal{B}_{2}=\{4567,1458,1678,3568,2478,3468,2568\}, \\
& \mathcal{B}_{3}=\{4567,2367,1678,3568,2478,3468,2568\}, \\
& \mathcal{B}_{4}=\{4567,2367,2345,3568,2478,3468,2568\} ;
\end{aligned}
$$

or is obtained by omitting one block either from the unique symmetric $(7,4,2)$ design or the unique symmetric $(7,3,1)$-design.

Proof (i) First, let $\mathcal{D}$ be nonprimary. This is the case only if $\lambda=1$, and so $k=2$, $v=4$. By Theorem $1, \mathcal{D}$ is obtained from a symmetric $(3,1,0)$-design by the technique described in Theorem 1. Applying this technique, it turns out that $\mathcal{D}$ is either the symmetric $(3,1,0)$-design with a point added to all of its blocks or the symmetric $(3,2,1)$-design with an extra isolated point. Now, let $\mathcal{D}$ be primary. In view of Theorem $4, \mathcal{D}$ is obtained by "pasting" two $\operatorname{BIBD}\left(b_{i}=v-1, v_{i}, r_{i}, k_{i}, \lambda_{i}\right)$ with $r_{i}=\lambda_{i}+1$. Keeping the notation of Theorem 4, we must have $f=1$. Thus $M$ is either the vector $\mathbf{0}$ or $\mathbf{1}$, and by Lemma 5, $N$ is the incidence matrix of either symmetric $(v-1,1,0)$-design or symmetric $(v-1, v-2, v-3)$-design.

(ii) First, let $\mathcal{D}$ be nonprimary. This is the case only if $\lambda=2$, and so $k=4, v=8$. By Theorem $1, \mathcal{D}$ is obtained from the Fano plane by the technique described in Theorem 1. Making use of the Maple procedure for checking graph isomorphism, it turns out that $\mathcal{D}$ is isomorphic to one of the pseudo designs $\mathcal{D}_{1}, \mathcal{D}_{2}, \mathcal{D}_{3}$, or $\mathcal{D}_{4}$. Now, let $\mathcal{D}$ be primary. Thus $\mathcal{D}$ is obtained by "pasting" two $\operatorname{BIBD}\left(\bar{b}_{i}=v-1, \bar{v}_{i}, \bar{r}_{i}, \bar{k}_{i}, \bar{\lambda}_{i}\right)$ 's with $\bar{r}_{i}=\bar{\lambda}_{i}+2$ for $i=1,2$. If $f=1$, then $M$ is either the vector $\mathbf{0}$ or $\mathbf{1}$, and by Lemma $5, N$ is the incidence matrix of either symmetric $(7,3,1)$-design or symmetric $(7,4,2)$-design. If $f \geq 2$, then $M$ and $N$ must be chosen from the incidence matrices of $\operatorname{BIBD}(6,4,3,2,1), \operatorname{BIBD}(6,3,3,2,2)$, or $\operatorname{BIBD}(2 \ell-2, \ell-1,2,1,0)$ for some $\ell \geq 2$. Since $\bar{v}_{1}+\bar{v}_{2}=v=\bar{b}_{1}+1=\bar{b}_{2}+1$, the only possible choices for $M$ and $N$ are that either

(1) $M$ is the incidence matrix of $\operatorname{BIBD}(6,4,3,2,1)$, and $N$ is that of $\operatorname{BIBD}(6,3,3$, $2,2)$; or

(2) $M$ is the incidence matrix of $\operatorname{BIBD}(6,4,3,2,1)$, and $N$ is that of $\operatorname{BIBD}(2 \ell-$ $2, \ell-1,2,1,0)$ for $\ell=3$.

If (1) is the case, then $v=7, s_{1}=3, s_{2}=4$, and so $3 k-5 \lambda=y=2$, which, together with $k-\lambda=2$, gives $\lambda=2$ and $k=4$. Now, $\mathcal{D}$ satisfies the conditions of parts 
(iii) and (iv) of Theorem 2. From part (iii) it follows that $\mathcal{D}$ is obtained from the symmetric $(7,4,2)$-design by omitting one of its blocks; and from part (iv) we see that $\mathcal{D}=\{3567,1467,1257,1236,2347,1345,2456\}$, which is again the symmetric $(7,4,2)$-design with an omitted block. If (2) is the case, then $(v, k, \lambda)=(7,3,1)$, and by Theorem 2(iii), $\mathcal{D}$ is obtained from the symmetric $(7,3,1)$-design by omitting one of its blocks.

Corollary 7 Conjecture 3 holds for pseudo $(v, k, \lambda)$-designs with $k=\lambda+1$ or $k=\lambda+2$.

\section{Graphs with many \pm 1 eigenvalues}

In this section we characterize all graphs of order $n$ whose spectrum contains a zero and \pm 1 with multiplicity $(n-3) / 2$. We show that this family of graphs consists of $\mathcal{S}_{2 k+1}, \mathcal{H}_{k, k+1}, \mathcal{R}_{2 k+1}, \mathcal{Q}_{2 k+1}$, where $n=2 k+1$, and two graphs of order 13 .

We begin by determining the spectrum of $\mathcal{S}_{n}, \mathcal{L}_{k, k}, \mathcal{H}_{k, k+1}, \mathcal{R}_{n}$, and $\mathcal{Q}_{n}$.

\section{Lemma 8}

(i) $\operatorname{spec}\left(\mathcal{S}_{2 k+1}\right)=\left\{ \pm \sqrt{k+1}, 0,( \pm 1)^{k-1}\right\}$,

(ii) $\operatorname{spec}\left(\mathcal{L}_{k, k}\right)=\left\{ \pm(k-1),( \pm 1)^{k-1}\right\}$,

(iii) $\operatorname{spec}\left(\mathcal{H}_{k, k+1}\right)=\left\{ \pm \sqrt{k^{2}-k+1}, 0,( \pm 1)^{k-1}\right\}$ for $k \geq 2$,

(iv) $\operatorname{spec}\left(\mathcal{R}_{2 k+1}\right)=\operatorname{spec}\left(\mathcal{Q}_{2 k+1}\right)=\left\{ \pm 2 \sqrt{k-2}, 0,( \pm 1)^{k-1}\right\}$ for $k \geq 3$.

Proof If one deletes the vertex of maximum degree from $\mathcal{S}_{2 k+1}$, what remain are $k$ copies of $K_{2}$. Thus, by interlacing, the spectrum of $\mathcal{S}_{2 k+1}$ contains \pm 1 of multiplicity at least $k-1$. Since $\mathcal{S}_{2 k+1}$ is a bipartite graph of an odd order, it has a zero eigenvalue. Let $\pm \theta$ be the remaining eigenvalues. As the sum of squares of eigenvalues of a graph is twice the number of edges, we have $2 \theta^{2}+2 k-2=4 k$, implying that $\theta=\sqrt{k+1}$. The spectrum of $\mathcal{L}_{k, k}$ is easily obtained since it has an adjacency matrix of the form

$$
\left(\begin{array}{cc}
O & J_{k}-I_{k} \\
J_{k}-I_{k} & O
\end{array}\right) .
$$

The graph $\mathcal{H}_{k, k+1}$ possesses an "equitable partition" with three cells in which each cell consists of the vertices with equal degree. (See [4, pp. 195-198] for more information on equitable partitions.) The adjacency matrix of the corresponding quotient is

$$
B=\left(\begin{array}{ccc}
0 & k-1 & 1 \\
k-1 & 0 & 0 \\
k & 0 & 0
\end{array}\right)
$$

with eigenvalues $\pm \sqrt{k^{2}-k+1}, 0$. Besides these three eigenvalues, by interlacing, $\mathcal{H}_{k, k+1}$ has \pm 1 eigenvalues of multiplicity at least $k-2$. Let $\pm \theta$ be the remaining 


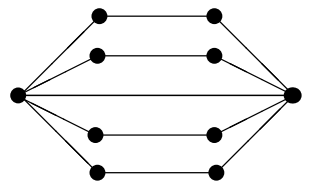

$G_{1}$

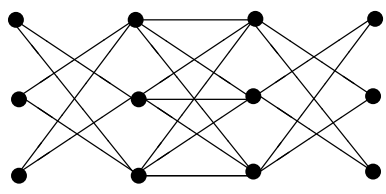

$G_{2}$

Fig. 2 The only nonregular graphs of order $n$ whose spectra contain $( \pm 1)^{\frac{n-2}{2}}$

eigenvalues. Thus, $2\left(k^{2}-k+1\right)+2(k-2)+2 \theta^{2}=2 k^{2}$, which implies $\theta=1$. If $N$ is the bipartite adjacency matrix of either $\mathcal{R}_{2 k+1}$ or $\mathcal{Q}_{2 k+1}$, then

$$
N N^{\top}-I=\left(\begin{array}{cc}
4 J_{k-3} & 2 J \\
2 J^{\top} & J_{3}
\end{array}\right)
$$

Thus $N N^{\top}-I$ is of rank one, and so both $\operatorname{spec}\left(\mathcal{R}_{2 k+1}\right)$ and $\operatorname{spec}\left(\mathcal{Q}_{2 k+1}\right)$ contain $\left\{0,( \pm 1)^{k-1}\right\}$. For the two remaining eigenvalues $\pm \theta$, we have the equation $2(k-1)+2 \theta^{2}=10(k-3)+12$, and so $\theta=2 \sqrt{k-2}$.

Before treating the graphs of the subject of this section, we deal with the graphs of order $n$ whose spectra contain $( \pm 1)^{\frac{n-2}{2}}$. If such a graph is regular, then it easily follows that $G$ must be $K_{\frac{n}{2}}, \frac{n}{2}$ minus a perfect matching. If it is regular, by [12, Proposition 8], $G$ is either the graph $G_{1}$ or $G_{2}$ of Fig. 2. So we have the following:

Theorem 9 (van Dam-Spence [12]) Let $G$ be a connected graph of order $n$. If the spectrum of $G$ contains $( \pm 1)^{\frac{n-2}{2}}$, then $G$ is either $\mathcal{L}_{\frac{n}{2}, \frac{n}{2}}$ or the graph $G_{1}$ or $G_{2}$ of Fig. 2.

Theorem 10 Let $G$ be a connected graph of order $n$. If the spectrum of $G$ contains $\left\{0,( \pm 1)^{\frac{n-3}{2}}\right\}$, then $G$ is one of the graphs $\mathcal{S}_{n}, \mathcal{R}_{n}, \mathcal{Q}_{n}, \mathcal{H}_{\frac{n-1}{2}, \frac{n+1}{2}}, G_{3}$, or $G_{4}$ of Fig. 3.

Proof From the spectrum of $G$ it is obvious that $G$ is bipartite of order $n=2 k+1$. Let $N$ be the $r \times s$ bipartite adjacency matrix of $G$ where $r+s=2 k+1$ and $r \leq s$. Considering the rank of the adjacency matrix of $G$, we have $\operatorname{rank}(N)=k$. This implies that $r=k$ and $s=k+1$. So $N N^{\top}$ is nonsingular with two distinct eigenvalues $1, \theta$, say. Since the multiplicity of eigenvalue 1 is $k-1, N N^{\top}-I$ is a rank-one matrix, and by the Perron-Frobenius theorem, one may choose a positive eigenvector $\mathbf{x}=\left(x_{1}, \ldots, x_{k}\right)$ of $N N^{\top}$ for $\theta$ so that

$$
N N^{\top}=I+\mathbf{x}^{\top} \mathbf{x} .
$$

If the vertices corresponding to the rows of $N$ are labeled $\{1, \ldots, k\}$, from (3) it follows that

$$
\begin{aligned}
d_{i} & =1+x_{i}^{2}, \\
d_{i j} & =x_{i} x_{j},
\end{aligned}
$$


Fig. 3 The graphs $G_{3}$ and $G_{4}$
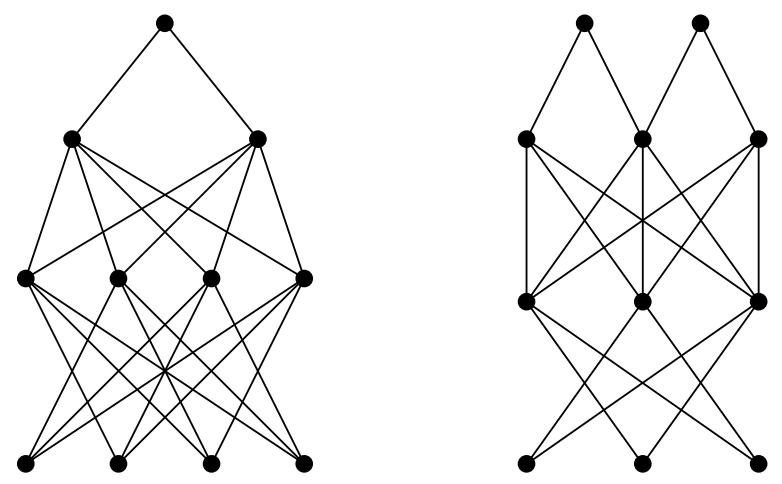

where $d_{i}$ and $d_{i j}$ for $i, j=1, \ldots, k$ are the degree of the vertex $i$ and the number of common neighbors of the vertices $i, j$, respectively. It turns out that $\mathbf{x}=\sqrt{\delta} \mathbf{w}$, where $\mathbf{w}=\left(w_{1}, \ldots, w_{k}\right)$ is a positive integer vector, and $\delta$ is a square-free integer.

First let $d_{i}=d$ for $i=1, \ldots, k$. By (4) and (5), $d_{i j}=d-1$ for all $i, j$. This means that $N$ is the incidence matrix of a pseudo $(k, d, d-1)$-design. Therefore from Theorem 6 it follows that $G$ is either $\mathcal{S}_{2 k+1}$ or $\mathcal{H}_{k, k+1}$.

Now let $d_{i}>d_{j}$ for some $i, j$. Thus $w_{i} \geq w_{j}+1$, and

$$
d_{j} \geq d_{i j}=\delta w_{i} w_{j} \geq \delta w_{j}^{2}+\delta w_{j} \geq \delta w_{j}^{2}+1=d_{j} .
$$

So one must have the equality in all the above inequalities which implies $\delta=w_{j}=1$, $w_{i}=2$, and so $d_{i j}=d_{j}=2, d_{i}=5$. Therefore, the vertices of $G$ corresponding to the rows of $N$ are of degree either 2 or 5, and any vertex of degree 2 has all of its neighbors in common with any vertex of degree 5. It thus follows that $N$ can be rearranged so that

$$
N=\left(\begin{array}{cc}
N_{1} & J \\
O & N_{2}
\end{array}\right),
$$

where $N_{1}$ and $N_{2}$ correspond to the vertices of degrees 5 and 2, respectively. Suppose that $N_{1}$ and $N_{2}$ are $k_{1} \times \ell_{1}$ and $k_{2} \times \ell_{2}$, respectively. With the above rearrangement, $\mathbf{x}=(2, \ldots, 2,1, \ldots, 1)$ with $k_{1} 2$ s and $k_{2} 1$ s. So

$$
\mathbf{x}^{\top} \mathbf{x}=\left(\begin{array}{cc}
4 J_{k_{1}, k_{1}} & 2 J_{k_{1}, k_{2}} \\
2 J_{k_{2}, k_{1}} & J_{k_{2}, k_{2}}
\end{array}\right) .
$$

If $\ell_{1}=0$, then

$$
N N^{\top}=\left(\begin{array}{c}
J \\
N_{2}
\end{array}\right)\left(J^{\top} N_{2}^{\top}\right)=\left(\begin{array}{cc}
\ell_{2} J_{k_{1}, k_{1}} & 2 J_{k_{1}, k_{2}} \\
2 J_{k_{2}, k_{1}} & J_{k_{2}, k_{2}}+I
\end{array}\right) .
$$

In view of (3), we must have $k_{1}=1$, which in turn implies that $\ell_{2}=5$ and $k_{2}=3$. So the graph $G$ consists of three vertices of degree 2 , say $v_{1}, v_{2}, v_{3}$, and one vertex of degree 5 in one part and five other vertices in the other part such that each of these five latter vertices is adjacent to at least one of $v_{1}, v_{2}, v_{3}$. On the other hand, 
since $N_{2} N_{2}^{\top}=J+I$, each pair of $v_{1}, v_{2}, v_{3}$ have a common neighbor, which is not possible. Therefore, $\ell_{1}>0$. By inspecting $N N^{\top}$ and (3) we have

$$
\begin{aligned}
& N_{1} N_{1}^{\top}=I+\left(4-\ell_{2}\right) J, \\
& N_{2} N_{2}^{\top}=I+J,
\end{aligned}
$$

and moreover, one of $N_{1}$ or $N_{2}$ must be square since otherwise from (6) it is clear that $\operatorname{rank}(N) \leq k-2$, which is a contradiction.

First let $N_{1}$ be square. Thus $N_{1}$ is the incidence matrix of a symmetric $\left(k_{1}, 3-\ell_{2}\right.$, $\left.4-\ell_{2}\right)$-design $\mathcal{D}_{1}$, and $N_{2}$ is the incidence matrix of a pseudo $\left(\ell_{2}, 2,1\right)$-design. Therefore, by Theorem $5, \mathcal{D}_{1}$ is either the symmetric $\left(k_{1}, 1,0\right)$-design or the symmetric $\left(k_{1}, k_{1}-1, k_{1}-2\right)$-design. If $\mathcal{D}_{1}$ is the symmetric $\left(k_{1}, 1,0\right)$-design, then $\ell_{2}=4$, and so by Theorem $6, \mathcal{D}_{2}$ is obtained from the symmetric $(3,1,0)$-design by adding a new point to all of its blocks. So we find that $N_{1}=I_{k_{1}}$ and $N_{2}=\widetilde{I}_{3}$. Therefore, $G$ is $\mathcal{R}_{n}$. If $\mathcal{D}_{2}$ is the symmetric $\left(k_{1}, k_{1}-1, k_{1}-2\right)$-design, then we must have $4-\ell_{2}=k_{1}-2$, and so $k_{2}=5-k_{1}$. As $I+\left(4-\ell_{2}\right) J$ is a positive semidefinite matrix, $\ell_{2} \leq 4$. As $k_{2} \geq 1$, we have also $\ell_{2} \geq 2$. If $\ell_{2}=4$, then $G$ is $\mathcal{R}_{11}$. If $\ell_{2}=2,3$, then $\left(k_{1}, k_{2}\right)=(4,1)$ or $(3,2)$, from which it follows that $G$ is either $G_{3}$ or $G_{4}$, respectively.

Now, let $N_{2}$ be square. From Theorem 5 it follows that $N_{2}$ is the incidence matrix of the symmetric $\left(k_{2}, k_{2}-1, k_{2}-2\right)$-design. By (8), $k_{2}-2=1$. Thus $N_{2}=J_{3}-I_{3}$, $\ell_{2}=3$, and $N_{1} N_{1}^{\top}=I+J$. So $N_{1}$ is the incidence matrix of a pseudo design, which by Theorem 6 can be obtained in one of the following three ways: (1) From a symmetric $\left(k_{1}, 1,0\right)$-design by adding an extra point to all the blocks, i.e., $N_{1}=\widetilde{I_{k_{1}}}$, which means that $G$ is the graph $\mathcal{Q}_{n}$. (2) From a symmetric $\left(k_{1}, k_{1}-1, k_{1}-2\right)$-design by adding an extra point to all the blocks, so $k_{1}-2=0$, which implies $G$ to be $\mathcal{Q}_{5}$. (3) From a symmetric $\left(k_{1}, k_{1}-1, k_{1}-2\right)$-design by adding an isolated point, which is impossible as this makes $G$ disconnected.

In the rest of this section we determine the spectral characterization of the graphs discussed so far. We begin by $\mathcal{L}_{k, k}$ which is readily seen to be DS as it is the only $(k-1)$-regular bipartite graph of order $2 k$.

For later use, we need to mention the spectrum of the graphs $G_{1}, G_{2}, G_{3}$, and $G_{4}$ :

$$
\begin{aligned}
& \operatorname{spec}\left(G_{1}\right)=\left\{ \pm 3,( \pm 1)^{4}\right\}, \quad \operatorname{spec}\left(G_{2}\right)=\left\{ \pm 4,( \pm 1)^{5}\right\} \\
& \operatorname{spec}\left(G_{3}\right)=\left\{ \pm 3 \sqrt{2}, 0,( \pm 1)^{4}\right\}, \quad \operatorname{spec}\left(G_{4}\right)=\left\{ \pm \sqrt{15}, 0,( \pm 1)^{4}\right\} .
\end{aligned}
$$

Corollary 11 The graph $\mathcal{H}_{k, k+1}$ is DS for $k \geq 2$.

Proof Any cospectral mate $H$ of $\mathcal{H}_{k, k+1}$ for $k \geq 2$ must have one of the graphs of Theorems 9 and 10 as a connected component. Noting that $k^{2}-k+1$ is always odd and never (unless $k=1$ ) a perfect square, we see that $H$ cannot have one of $\mathcal{L}_{t, t}$, $\mathcal{R}_{2 t+1}, \mathcal{Q}_{2 t+1}$ for any $t$ or $G_{1}, G_{2}, G_{3}$ as a component. Considering the number of edges, we see that $\mathcal{S}_{2 t+1}$ for any $t$ cannot be a component of $H$. The same is for $G_{4}$ as the equation $k^{2}-k+1=15$ has no integral solution. 
The graphs $\mathcal{S}_{n}$ belong to a family of trees called starlike trees (trees with only one vertex of degree larger than 2). In [11], it was asked to determine which starlike trees are DS. Partial results are obtained by several authors (cf. [11]). For this specific starlike trees, Brouwer [2] showed that the graphs $\mathcal{S}_{n}$ are DS among trees. Here, we completely determine the spectral characterization of the graphs $\mathcal{S}_{n}$. The proof is the same as the proof of the above corollary.

Corollary 12 The graph $\mathcal{S}_{2 k+1}$ is DS if $k \notin S$, where

$$
S=\{4 \ell+3 \mid \ell \in \mathbb{N}\} \cup\left\{\ell^{2}-1 \mid \ell \in \mathbb{N}\right\} \cup\left\{\ell^{2}-\ell \mid \ell \in \mathbb{N}\right\} \cup\{14,17\} .
$$

Moreover, for $k \in S$, we have:

- $\mathcal{S}_{17}$ has exactly two cospectral mates, which are $\mathcal{L}_{4,4} \cup 4 K_{2} \cup K_{1}$ and $G_{1} \cup$ $3 K_{2} \cup K_{1}$;

- $\mathcal{S}_{29}$ has exactly one cospectral mate, which is $G_{4} \cup 9 K_{2}$;

- $\mathcal{S}_{31}$ has exactly four cospectral mates, which are $G_{2} \cup 9 K_{2} \cup K_{1}, \mathcal{L}_{5,5} \cup 10 K_{2} \cup K_{1}$, $\mathcal{R}_{13} \cup 9 K_{2}$, and $\mathcal{Q}_{13} \cup 9 K_{2}$;

- $\mathcal{S}_{35}$ has exactly one cospectral mates, which is $G_{3} \cup 12 K_{2}$;

- if $k=4 \ell+3$ and $\ell$ is not an integer of the form $t^{2}-1$, then $\mathcal{S}_{2 k+1}$ has exactly two cospectral mates, which are $\mathcal{R}_{2 \ell+7} \cup 3 \ell K_{2}$ and $\mathcal{Q}_{2 \ell+7} \cup 3 \ell K_{2}$;

- if $k=\ell^{2}-1, \ell=2 t$, and $k \neq 15$, then $\mathcal{S}_{2 k+1}$ has exactly three cospectral mates, which are $\mathcal{L}_{\ell+1, \ell+1} \cup(k-\ell-1) K_{2} \cup K_{1}, \mathcal{R}_{2 t^{2}+5} \cup 3\left(t^{2}-1\right) K_{2}$, and $\mathcal{Q}_{2 t^{2}+5} \cup$ $3\left(t^{2}-1\right) K_{2}$;

- if $k=\ell^{2}-1, \ell$ is odd, and $k \neq 8$, then $\mathcal{S}_{2 k+1}$ has exactly one cospectral mate, which is $\mathcal{L}_{\ell+1, \ell+1} \cup(k-\ell-1) K_{2} \cup K_{1}$;

- if $k=\ell^{2}-\ell$, then $\mathcal{S}_{2 k+1}$ has exactly one cospectral mate, which is $\mathcal{H}_{\ell, \ell+1} \cup$ $(k-\ell) K_{2}$.

Corollary 13 The graph $\mathcal{R}_{7}$ has exactly three cospectral mates, namely $\mathcal{Q}_{7}, S_{7}$, and $\mathcal{L}_{3,3} \cup K_{1}$. If $k=\ell^{2}+2$, for some $\ell \geq 2$, then the graph $\mathcal{R}_{2 k+1}$ has exactly two cospectral mates, namely $\mathcal{Q}_{2 k+1}$ and $\mathcal{L}_{2 \ell+1,2 \ell+1} \cup(\ell-1)^{2} K_{2} \cup K_{1}$. For other values of $k \geq 3$, the only cospectral mate of $\mathcal{R}_{2 k+1}$ is $\mathcal{Q}_{2 k+1}$.

In addition to that the graphs $\mathcal{R}_{n}$ and $\mathcal{Q}_{n}$ are cospectral, they are related through switching. We first recall the Seidel switching. Let $G$ be a graph with vertex set $V$, and $X \subseteq V$. From $G$ we obtain a new graph by leaving adjacency and nonadjacency inside $X$ and $V \backslash X$ as it was, and interchanging adjacency and nonadjacency between $X$ and $V \backslash X$. This new graph is said to be obtained by Seidel switching with respect to the set $X$. Now, in the graph $\mathcal{R}_{n}$, let $X$ be the set of four vertices corresponding to the columns of the submatrix $J$ in the bipartite adjacency matrix of $\mathcal{R}_{n}$. If we apply the Seidel switching on $\mathcal{R}_{n}$ with respect to $X$, we obtain $\mathcal{Q}_{n}$.

\section{Graphs with many $\pm \sqrt{2}$ eigenvalues}

In this section we characterize all graphs of order $n$ whose spectra contain a zero and $\pm \sqrt{2}$ with multiplicity $(n-3) / 2$. It turns out that, up to isomorphism, there are exactly six such graphs, all of which are obtained in some way from the Fano plane. 
We start with graphs of order $n$ whose spectra contain $( \pm \sqrt{2})^{\frac{n-2}{2}}$. Let $N$ be the $\frac{n}{2} \times \frac{n}{2}$ bipartite adjacency matrix of $G$. If $G$ is regular of degree $k$, say, then $N N^{\top}=$ $(k-2) I+2 J$, which means that $N$ is the incidence matrix of an $(n / 2, k, k-2)$ design. Hence, by Lemma 5, $N$ is the incidence matrix of either the Fano plane or the complement of the Fano plane. The nonregular ones are characterized in [12, Proposition 9].

Theorem 14 (van Dam-Spence [12]) Let $G$ be a connected graph of order $n$. If the spectrum of $G$ contains $( \pm \sqrt{2})^{\frac{n-2}{2}}$, then the bipartite adjacency matrix of $G$ is one of the following:

(i) incidence matrix of the Fano plane (i.e., G is the Heawood graph);

(ii) incidence matrix of the complement of the Fano plane;

(iii)

$$
\left(\begin{array}{cc}
N_{1} & J_{7} \\
O_{7} & N_{2}
\end{array}\right) \quad \text { or } \quad\left(\begin{array}{ccc}
1 & \mathbf{1}^{\top} & \mathbf{1}^{\top} \\
\mathbf{1} & I_{5} & I_{5} \\
\mathbf{1} & I_{5} & J_{5}-I_{5}
\end{array}\right),
$$

where $N_{1}$ and $N_{2}$ are the incidence matrices of the Fano plane and the symmetric $(7,4,2)$-design, respectively.

Theorem 15 Let $G$ be a connected graph of order $n$. If the spectrum of $G$ contains $\left\{0,( \pm \sqrt{2})^{\frac{n-3}{2}}\right\}$, then $G$ is incidence graph of one of

(i) the pseudo $(7,3,1)$-design;

(ii) the pseudo $(7,4,2)$-design; or

(iii) $\mathcal{D}_{1}, \ldots, \mathcal{D}_{4}$ of Theorem 6.

Proof From the spectrum of $G$ it is clear that $G$ is bipartite of order $n=2 k+1$. Let $N$ and $\mathbf{x}=\left(x_{1}, \ldots, x_{k}\right)$ be the same as in the proof of Theorem 10. Thus $N N^{\top}=$ $2 I+\mathbf{x}^{\top} \mathbf{x}$, and so

$$
\begin{aligned}
d_{i} & =2+x_{i}^{2}, \\
d_{i j} & =x_{i} x_{j} .
\end{aligned}
$$

Again we have $\mathbf{x}=\sqrt{\delta} \mathbf{w}$, where $\mathbf{w}=\left(w_{1}, \ldots, w_{k}\right)$ is a positive integer vector, and $\delta$ is a square-free integer.

First assume that there exist some $i, j$ such that $d_{i}>d_{j}$. Then $w_{i} \geq w_{j}+1$, and

$$
d_{j} \geq d_{i j}=\delta w_{i} w_{j} \geq \delta w_{j}^{2}+\delta w_{j} .
$$

If $\delta w_{j}=1$, then $\delta=w_{j}=1$, and so $d_{j}=2$, which is impossible. So $\delta w_{j}=2$, and equalities must occur in all the above inequalities. Hence two cases may occur: (1) $\delta=1$ and $w_{j}=2$, which implies $d_{j}=d_{i j}=6$ and $d_{i}=11$; or (2) $\delta=2$ and $w_{j}=1$, which implies $d_{j}=d_{i j}=4$ and $d_{i}=10$. Again, like in the proof of Theorem 10, $N$ can be rearranged so that

$$
N=\left(\begin{array}{cc}
N_{1} & J \\
O & N_{2}
\end{array}\right),
$$


where $N_{2}$ with $k_{2}$ rows, say, correspond to the vertices with smaller degrees. Then in the same manner as in the proof of Theorem 10, we see that $N_{2} N_{2}^{\top}=2 I+\ell J$, where $\ell$ is either 16 or 36 . As $N_{2}$ is either $k_{2} \times k_{2}$ or $k_{2} \times\left(k_{2}+1\right)$, it is the incidence matrix of either a symmetric $\left(k_{2}, \ell+2, \ell\right)$-design or a pseudo $\left(k_{2}, \ell+2, \ell\right)$-design. Such designs do not exist by Lemma 5 and Theorem 6.

Therefore, $d_{i}=d$ for $i=1, \ldots, k$. By (10) and (11), $d_{i j}=d-2$ for all $i, j$. So $N$ is the incidence matrix of a pseudo $(k, d, d-2)$-design. Thus the result follows from Theorem 6.

Acknowledgements The research of the author was in part supported by a grant from IPM (No. 90050117). The author is grateful to Osvaldo Marrero for supplying the literature of pseudo designs, to Jack Koolen for carefully reading the manuscript, and to the referees whose helpful comments improved the presentation of the paper.

\section{References}

1. Bose, R.C., Shrikhande, S.S., Singhi, N.M.: Edge regular multigraphs and partial geometric designs with an application to the embedding of quasi-regular designs. In: Colloquio Internazionale sulle Teorie Combinatorie, Tomo I, Rome, 1973. Atti dei Convegni Lincei, vol. 17, pp. 49-81. Accad. Naz. Lincei, Rome (1976)

2. Brouwer, A.E.: Small integral trees. Electron. J. Comb. 15, Note 1 (2008)

3. Cameron, P.J.: Research problems from the 19th British Combinatorial Conference. Discrete Math. 293, 313-320 (2005)

4. Godsil, C., Royle, G.: Algebraic Graph Theory. Springer, New York (2001)

5. Marrero, O.: Completion and embedding between pseudo $(v, k, \lambda)$-designs and $(v, k, \lambda)$-designs. Bull. Am. Math. Soc. 80, 103-105 (1974)

6. Marrero, O.: A survey of pseudo $(v, k, \lambda)$-designs. Aequ. Math. 16, 195-220 (1977)

7. Marrero, O., Butson, A.T.: Modular Hadamard matrices and related designs. J. Comb. Theory, Ser. A 15, 257-269 (1973)

8. Ryser, H.J.: An extension of a theorem of de Bruijn and Erdős on combinatorial designs. J. Algebra 10, 246-261 (1968)

9. Ryser, H.J.: Symmetric designs and related configurations. J. Comb. Theory, Ser. A 12, 98-111 (1972)

10. van Dam, E.R., Haemers, W.H.: Which graphs are determined by their spectrum? Linear Algebra Appl. 373, 241-272 (2003)

11. van Dam, E.R., Haemers, W.H.: Developments on spectral characterizations of graphs. Discrete Math. 309, 576-586 (2009)

12. van Dam, E.R., Spence, E.: Combinatorial designs with two singular values-I: uniform multiplicative designs. J. Comb. Theory, Ser. A 107, 127-142 (2004)

13. van Dam, E.R., Spence, E.: Combinatorial designs with two singular values II. Partial geometric designs. Linear Algebra Appl. 396, 303-316 (2005)

14. Woodall, D.R.: Square $\lambda$-linked designs. Proc. Lond. Math. Soc. 20, 669-687 (1970) 